\title{
New Nonlinear Systems Admitting Virasoro-Type Symmetry Algebra and Group-Invariant Solutions
}

\author{
Lizhen Wang, ${ }^{1,2}$ Qing Huang, ${ }^{1,2}$ and Yanmei $\mathrm{Di}^{3}$ \\ ${ }^{1}$ Center for Nonlinear Studies, Northwest University, Xi'an 710069, China \\ ${ }^{2}$ Department of Mathematics, Northwest University, Xian 710069, China \\ ${ }^{3}$ Department of Applied Mathematics, Zhejiang University of Technology, Hangzhou 310023, China
}

Correspondence should be addressed to Lizhen Wang; wanglz123@hotmail.com

Received 28 December 2013; Accepted 20 January 2014; Published 3 March 2014

Academic Editor: Chaudry Masood Khalique

Copyright (C) 2014 Lizhen Wang et al. This is an open access article distributed under the Creative Commons Attribution License, which permits unrestricted use, distribution, and reproduction in any medium, provided the original work is properly cited.

With the aid of symbolic computation by Maple, we extend the application of Virasoro-type symmetry prolongation method to coupled systems with two-component nonlinear equations. New nonlinear systems admitting infinitely dimensional centerless Virasoro-type symmetry algebra are constructed. Taking one of them as an example, we present some group-invariant solutions to one of the new model systems.

\section{Introduction}

Integrable models such as the KdV equation, the KP equation, the nonlinear Schrödinger equation, the NNV equation, the sine-Gordon equation, and the Toda lattice have played more and more important roles in almost all natural sciences. It becomes one of the most fundamental problems to seek for as much as possible new nonlinear equations and systems with some nice properties including Lax pair, Painlevé property, infinite number of conservation laws, and bi-Hamiltonian structures.

There exist many powerful methods to construct nonlinear equations and systems like the multiscale method, symmetry constraint method, and conformal invariant method [1-4]. Among these methods developed recently, the Virasoro-type symmetry prolongation (VSP) method is found to be very effective. Based on the fact that all the known $(2+1)$-dimensional integrable models possess the following centerless Virasoro-type subalgebra:

$$
\left[\sigma\left(f_{1}\right), \sigma\left(f_{2}\right)\right]=\sigma\left(\dot{f}_{1} f_{2}-\dot{f}_{2} f_{1}\right)
$$

where $f_{1}$ and $f_{2}$ are arbitrary functions of the same argument and there are no known nonintegrable models owning such type symmetry algebra, Lou and $\mathrm{Hu}$ introduced an idea that if an $f$-independent model possesses the Virasoro-type symmetry algebra (1), the model is Virasoro integrable [5]. By using this theory and selecting the special realizations, some new $(2+1)$-dimensional and $(3+1)$-dimensional Virasoro integrable models have been derived [6-8].

However, the VSP method and concrete realizations discussed above all belong to single equations. To our knowledge, there are few results concerning the construction of coupled systems with two-component nonlinear equations [9]. Therefore we extend the applications of this method to construct several $(2+1)$-dimensional Virasoro integrable systems by selecting special realization of algebra (1).

The remainder of this paper is organized as follows. The general theory of the VSP method for nonlinear systems is presented in Section 2. In Section 3, some (2+1)-dimensional Virasoro integrable systems are constructed by choosing appropriate realizations. For a concrete example, the onedimensional optimal system and group-invariant solutions to system (21) are given in Section 4. The last section contains some concluding remarks. 


\section{The Generalized VSP Method}

Firstly, let us give a brief account of the generalized VSP method for nonlinear systems. We consider the vector field with the following form:

$$
\begin{aligned}
\vec{V}= & X(x, y, t, u, v) \partial_{x}+Y(x, y, t, u, v) \partial_{y}+T(x, y, t, u, v) \partial_{t} \\
& +U(x, y, t, u, v) \partial_{u}+V(x, y, t, u, v) \partial_{v} .
\end{aligned}
$$

We define the functions $T, X, Y, U$, and $V$ as follows:

$$
\begin{gathered}
T=f(t), \\
\{X, Y, U, V\}=\left\{\sum_{i=1}^{n} f^{(i)} X_{i}, \sum_{i=1}^{n} f^{(i)} Y_{i}, \sum_{i=1}^{n} f^{(i)} U_{i}, \sum_{i=1}^{n} f^{(i)} V_{i}\right\}, \\
n=1,2,3, \ldots,
\end{gathered}
$$

where $f^{(i)}=\mathrm{d}^{i} f / \mathrm{d} t^{i}, X_{i}, Y_{i}, U_{i}, V_{i}, i=1,2,3, \ldots$, are functions of the variables $\{x, y, t, u, v\}$ and should be selected to satisfy the commutation relation (1). In order to construct invariant $k$ th-order partial differential equations, we should calculate the $k$ th prolongation of the vector field $\vec{V}$ firstly. The general formula for the $k$ th prolongation of a vector field $\vec{V}$ is given by

$$
\begin{aligned}
p r^{(k)} \vec{V}= & \vec{V}+U^{x} \partial_{u_{x}}+U^{y} \partial_{u_{y}}+U^{t} \partial_{u_{t}}+V^{x} \partial_{v_{x}} \\
& +V^{y} \partial_{v_{y}}+V^{t} \partial_{v_{t}}+\cdots+\sum_{1 \leq i+j+l \leq k} U^{x^{i} y^{j} t^{l}} \partial_{u_{x^{i} y_{t} l^{l}}} \\
& +\sum_{1 \leq i+j+l \leq k} V^{x^{i} y^{j} t^{l}} \partial_{v_{x^{i} y^{j}} l},
\end{aligned}
$$

with

$$
\begin{aligned}
U^{x^{i} y^{j} t^{l}} & \\
= & D_{x} U^{x^{i-1} y^{j} t^{l}}-\left(D_{x} X\right) u_{x^{i} y^{j} t^{l}} \\
& -\left(D_{x} Y\right) u_{x^{i-1} y^{j+1} t^{l}}-\left(D_{x} T\right) u_{x^{i-1} y^{j} t^{l+1}} \\
= & D_{y} U^{x^{i} y^{j-1} t^{l}}-\left(D_{y} X\right) u_{x^{i+1} y^{j-1} t^{l}} \\
& -\left(D_{y} Y\right) u_{x^{i} y^{j} t^{l}}-\left(D_{y} T\right) u_{x^{i} y^{j-1} t^{l+1}} \\
= & D_{t} U^{x^{i} y^{j} t^{l-1}}-\left(D_{t} X\right) u_{x^{i+1} y^{j} t^{l-1}} \\
& -\left(D_{t} Y\right) u_{x^{i} y^{j+1} t^{l-1}}-\left(D_{t} T\right) u_{x^{i} y^{j} t^{l}}
\end{aligned}
$$

$$
\begin{aligned}
& V^{x^{i} y^{j}} t^{l} \\
&=D_{x} V^{x^{i-1} y^{j} t^{l}}-\left(D_{x} X\right) v_{x^{i} y^{j} t^{l}} \\
&-\left(D_{x} Y\right) v_{x^{i-1} y^{j+1} t^{l}}-\left(D_{x} T\right) v_{x^{i-1} y^{j} t^{l+1}} \\
&= D_{y} V^{x^{i} y^{j-1} t^{l}}-\left(D_{y} X\right) v_{x^{i+1} y^{j-1} t^{l}} \\
&-\left(D_{y} Y\right) v_{x^{i} y^{j} t^{l}-\left(D_{y} T\right) v_{x^{i} y^{j-1}} t^{l+1}} \\
&= D_{t} V^{x^{i} y^{j} t^{l-1}}-\left(D_{t} X\right) v_{x^{i+1} y^{j} t^{l-1}} \\
&-\left(D_{t} Y\right) v_{x^{i} y^{j+1} t^{l-1}}-\left(D_{t} T\right) v_{x^{i} y^{j} t^{l},}
\end{aligned}
$$

where $D_{x}, D_{y}$, and $D_{t}$ are total derivatives with respect to $x, y$, $t$, respectively. Thus we can calculate the $k$ th prolongation of a concrete vector $\vec{V}$. It is well known that the invariant system should have the following form:

$$
\Delta\left(x, y, t, u, v, u_{x}, v_{x}, u_{y}, v_{y}, u_{t}, v_{t}, \ldots, u_{x^{i} y^{j} t^{l}}, v_{x^{i} y^{j} t^{l}}, \ldots\right)=0
$$

where $\Delta$ satisfies

$$
\left.\operatorname{pr}^{(k)} \vec{V}(\Delta)\right|_{\Delta=0}=0
$$

In order to construct group invariant equations, we should solve the corresponding characteristic equations

$$
\begin{aligned}
\frac{\mathrm{d} t}{T} & =\frac{\mathrm{d} x}{X}=\frac{\mathrm{d} y}{Y}=\frac{\mathrm{d} u}{U}=\frac{\mathrm{d} v}{V} \\
& =\cdots=\frac{\mathrm{d} u_{x^{i} y^{j} t^{l}}}{U^{x^{i} y^{j} t^{l}}}=\frac{\mathrm{d} v_{x^{i} y^{j} t^{l}}}{V^{x^{i} y^{j} t^{l}}}=\cdots .
\end{aligned}
$$

After solving the above system, we can obtain a set of elementary invariants

$$
\begin{aligned}
& I_{m}\left(x, y, t, u, v, \ldots, u_{x^{i} y^{j} t^{l}}, v_{x^{i} y^{j} t^{l}}\right) \\
& \quad \equiv I_{m} \quad(1 \leq i+j+l \leq k, m=1,2,3, \ldots) .
\end{aligned}
$$

The general $\vec{V}$ invariant system has the following form:

$$
\begin{aligned}
& H_{1}\left(I_{1}, I_{2}, I_{3}, \ldots, I_{r}, \ldots\right)=0 \\
& H_{2}\left(I_{1}, I_{2}, I_{3}, \ldots, I_{r}, \ldots\right)=0
\end{aligned}
$$

According to the definition of the Virasoro integrability, the model should be $f$-independent. Therefore, when we find out the $f$-independent group invariants, we can construct the new Virasoro integral models from (10). Compared with the VSP method (see [7]), this method can be used to deal with coupled systems with two-component nonlinear equations. 


\section{Applications}

In this section, we will construct several coupled systems admitting Virasoro-type symmetry algebra by selecting concrete realization of (1). The realization we consider is

$$
\begin{aligned}
\vec{V}= & f \partial_{t}+C_{1} \dot{f} x \partial_{x}+C_{2} \dot{f} y \partial_{y} \\
& +C_{3} \dot{f} p \partial_{p}+\left(C_{4} \dot{f} r+C_{5} \ddot{f} x\right) \partial_{r},
\end{aligned}
$$

where $\dot{f}, \ddot{f}$, and $\dddot{f}$ denote the first, second, and third order derivatives of function $f=f(t)$ with respect to $t$, respectively, and $C_{i}, i=1, \ldots, 5$, are arbitrary constants. It is easy to verify that $\vec{V}$ is a Virasoro type symmetry when $C_{1}-C_{4}=1$. According to the prolongation formula (4), one can obtain the corresponding $k$ th prolongation of $\vec{V}$ with the aid of symbolic computation by Maple:

$$
\begin{aligned}
& \operatorname{pr}^{(k)} \vec{V} \\
& =\vec{V}+\left(C_{3}-C_{1}\right) \dot{f} p_{x} \partial_{p_{x}}-\left(\dot{f} r_{x}-C_{5} \ddot{f}\right) \partial_{r_{x}} \\
& +\left(C_{3}-C_{2}\right) \dot{f} p_{y} \partial_{p_{y}}+\left(C_{4}-C_{2}\right) \dot{f} r_{y} \partial_{r_{y}} \\
& +\left[\left(C_{3}-1\right) \dot{f} p_{t}+C_{3} p \ddot{f}-C_{1} \ddot{f} x p_{x}-C_{2} \ddot{f} y p_{y}\right] \partial_{p_{t}} \\
& +\left[\left(C_{4}-1\right) \dot{f} r_{t}+C_{4} \ddot{f} r+C_{5} \dddot{f} x-C_{1} \ddot{f} x r_{x}-C_{2} \ddot{f} y r_{y}\right] \\
& \times \partial_{r_{t}}+\left(C_{3}-2 C_{1}\right) \dot{f} p_{x x} \partial_{p_{x x}}-\left(1+C_{1}\right) \dot{f} r_{x x} \partial_{r_{x x}} \\
& +\left(C_{3}-C_{2}-C_{1}\right) \dot{f} p_{x y} \partial_{p_{x y}}-\left(1+C_{2}\right) \dot{f} r_{x y} \partial_{r_{x y}} \\
& +\left[\left(C_{3}-C_{1}-1\right) \dot{f} p_{x t}+\left(C_{3}-C_{1}\right) \ddot{f} p_{x}\right. \\
& \left.-C_{1} \ddot{f} x p_{x x}-C_{2} \ddot{f} y p_{x y}\right] \partial_{p_{x t}} \\
& +\left[-2 \dot{f} r_{x t}-\ddot{f} r_{x}+C_{5} \dddot{f}-C_{1} \ddot{f} x r_{x x}-C_{2} \ddot{f} y r_{x y}\right] \partial_{r_{x t}} \\
& +\left(C_{3}-2 C_{2}\right) p_{y y} \dot{f} \partial_{p_{y y}}+\left(C_{4}-2 C_{2}\right) r_{y y} \dot{f} \partial_{r_{y y}} \\
& +\left[\left(C_{3}-C_{2}-1\right) \dot{f} p_{y t}+\left(C_{3}-C_{2}\right) \ddot{f} p_{y}\right. \\
& \left.-C_{1} \ddot{f} x p_{x y}-C_{2} \ddot{f} y p_{y y}\right] \partial_{p_{y t}} \\
& +\left[\left(C_{4}-C_{2}-1\right) \dot{f} r_{y t}+\left(C_{4}-C_{2}\right) \ddot{f} r_{y}\right. \\
& \left.-C_{1} x \ddot{f} r_{x y}-C_{2} \ddot{f} y r_{y y}\right] \partial_{r_{y t}} \\
& +\left[\left(C_{3}-2\right) \dot{f} p_{t t}+\left(2 C_{3}-1\right) \ddot{f} p_{t}+C_{3} p \dddot{f}-C_{1} x \dddot{f} p_{x}\right. \\
& \left.-2 C_{1} x \ddot{f} p_{x t}-C_{2} y \dddot{f} p_{y}-2 C_{2} y \ddot{f} p_{y t}\right] \partial_{p_{t t}} \\
& +\left[\left(C_{4}-2\right) \dot{f} r_{t t}+\left(2 C_{4}-1\right) \ddot{f} r_{t}+C_{4} r \ddot{f}+C_{5} x f^{(4)}\right. \\
& \left.-C_{1} x \dddot{f} r_{x}-2 C_{1} x \ddot{f} r_{x t}-C_{2} y \dddot{f} r_{y}-2 C_{2} y \ddot{f} r_{y t}\right] \partial_{r_{t t}}
\end{aligned}
$$

$$
\begin{aligned}
& +\left(C_{3}-3 C_{1}\right) \dot{f} p_{x x x} \partial p_{x x x}+\left(C_{4}-3 C_{1}\right) \dot{f} r_{x x x} \partial_{r_{x x x}} \\
& +\left(C_{3}-2 C_{1}-C_{2}\right) \dot{f} p_{x x y} \partial_{p_{x x y}} \\
& +\left(C_{4}-2 C_{1}-C_{2}\right) \dot{f} r_{x x y} \partial_{r_{x x y}} \\
& +\left[\left(C_{3}-2 C_{1}\right) \ddot{f} p_{x x}+\left(C_{3}-2 C_{1}-1\right) \dot{f} p_{x x t}\right. \\
& \left.\quad-C_{1} x \ddot{f} p_{x x x}-C_{2} y \ddot{f} p_{x x y}\right] \partial_{p_{x x t}} \\
& +\left[\left(C_{4}-2 C_{1}\right) \ddot{f} r_{x x}+\left(C_{4}-2 C_{1}-1\right) \dot{f} r_{x x t}\right. \\
& \left.-C_{1} x \ddot{f} r_{x x x}-C_{2} y \ddot{f} r_{x x y}\right] \partial_{r_{x x t}}+\cdots .
\end{aligned}
$$

The corresponding characteristic equations of $p r^{(k)} \vec{V}$ are

$$
\begin{aligned}
\frac{\mathrm{d} t}{f} & =\frac{\mathrm{d} x}{C_{1} \dot{f} x}=\frac{\mathrm{d} y}{C_{2} \dot{f} y}=\frac{\mathrm{d} p}{C_{3} p \dot{f}}=\frac{\mathrm{d} r}{C_{4} r \dot{f}+C_{5} x \ddot{f}} \\
& =\cdots=\frac{\mathrm{d} u_{x^{i} y^{j} t^{r}}}{U^{x^{i} y^{j} t^{r}}}=\frac{\mathrm{d} v_{x^{i} y^{j} t^{r}}}{V^{x^{i} y^{j} t^{r}}} .
\end{aligned}
$$

After solving the above characteristic equations, we can obtain the explicit elementary invariants of $\vec{V}$ and some of them are listed as follows:

$$
\begin{aligned}
& I_{1}=x f^{-C_{1}}, \quad I_{2}=y f^{-C_{2}}, \quad I_{3}=p f^{-C_{3}} \text {, } \\
& I_{4}=r f^{-C_{4}}-C_{5} I_{1} \dot{f}, \quad I_{5}=p_{x} f^{-\left(C_{3}-C_{1}\right)}, \\
& I_{6}=r_{x} f-C_{5} \dot{f}, \quad I_{7}=p_{y} f^{-\left(C_{3}-C_{2}\right)}, \\
& I_{8}=r_{y} f^{-\left(C_{4}-C_{2}\right)}, \\
& I_{9}=p_{t} f^{1-C_{3}}-C_{3} I_{3} \dot{f}+C_{1} I_{1} I_{5} \dot{f}+C_{2} I_{2} I_{7} \dot{f}, \\
& I_{10}=r_{t} f^{1-C_{4}}-C_{5} I_{4} \dot{f}-\frac{1}{2} C_{5}^{2} I_{1}(\dot{f})^{2} \\
& +C_{5} I_{1}\left(f \ddot{f}-\frac{1}{2}(\dot{f})^{2}\right)-C_{1} I_{1} I_{6} \dot{f} \\
& -C_{2} I_{2} I_{8} \dot{f}-\frac{1}{2} C_{1} I_{1} C_{5} \dot{f}^{2}, \\
& I_{11}=p_{x x} f^{-\left(C_{3}-2 C_{1}\right)}, \quad I_{12}=r_{x x} f^{1+C_{1}}, \\
& I_{13}=p_{x y} f^{-\left(C_{3}-C_{2}-C_{1}\right)}, \quad I_{14}=r_{x y} f^{1+C_{2}} \text {, } \\
& I_{15}=p_{x t} f^{1+C_{1}-C_{3}}-\left(\left(C_{3}-C_{1}\right) I_{5}-C_{1} I_{1} I_{11}-C_{2} I_{2} I_{13}\right) \dot{f}, \\
& I_{16}=r_{x t} f^{2}+I_{6} \dot{f}+\frac{1}{2} C_{5}(\dot{f})^{2} \\
& +C_{5}\left(f \ddot{f}-\frac{1}{2}(\dot{f})^{2}\right)-C_{1} I_{1} I_{12} \dot{f}-C_{2} I_{2} I_{14} \dot{f} \\
& I_{17}=f^{2 C_{2}-C_{3}} p_{y y}, \quad I_{18}=f^{2 C_{2}-C_{4}} r_{y y} \text {, }
\end{aligned}
$$




$$
\begin{gathered}
I_{19}=f^{1+C_{2}-C_{3}} p_{y t}-\left(C_{3}-C_{2}\right) I_{7} \dot{f}+C_{1} I_{1} I_{13} \dot{f}+C_{2} I_{2} I_{17} \dot{f}, \\
I_{20}=r_{y t} f^{1+C_{2}-C_{4}}-\left[\left(C_{4}-C_{2}\right) I_{8}-C_{1} I_{1} I_{14}-C_{2} I_{2} I_{18}\right] \dot{f}, \\
I_{21}=p_{x x x} f^{3 C_{1}-C_{3}}, \quad I_{22}=r_{x x x} f^{3 C_{1}-C_{4}}, \\
I_{23}=p_{x x y} f^{2 C_{1}+C_{2}-C_{3}}, \quad I_{24}=r_{x x y} f^{2 C_{1}+C_{2}-C_{4}} .
\end{gathered}
$$

Substituting the above invariants into (10), one can establish various $(2+1)$-dimensional nonlinear systems. Generally speaking, it is difficult to find out all of the $f$-independent invariant systems. Here we only list some concrete examples.

Case 1. When selecting $C_{1}=1 / 3, C_{2}=0, C_{3}=-1 / 3, C_{4}=$ $-2 / 3$, and $C_{5}=-1 / 9$, we obtain the following group invariant system:

$$
\begin{gathered}
H_{1} \equiv I_{9}+I_{21}-3\left(I_{4} I_{5}+I_{3} I_{6}\right)+k_{1} I_{12}+k_{2} I_{13}^{2}=0, \\
H_{2} \equiv I_{8}+k_{3} I_{5}+k_{4} I_{17}^{2}+k_{5} I_{18}=0 .
\end{gathered}
$$

Here and hereafter $k_{i}, i=1, \ldots, 5$, are arbitrary constants. From the above invariant system, we deduce the corresponding Virasoro $f$-independent integrable system:

$$
\begin{gathered}
p_{t}+p_{x x x}-3 r p_{x}-3 p r_{x}+k_{1} r_{x x}+k_{2} p_{x y}^{2}=0, \\
r_{y}+k_{3} p_{x}+k_{4} p_{y y}^{2}+k_{5} r_{y y}=0 .
\end{gathered}
$$

Taking $k_{i}=0, i=1,2,4,5$, and $k_{3}=-1$, the above system is changed to be the asymmetry NNV equation which is considered as a model for an incompressible fluid and where $p$ and $r$ are the components of the velocity.

Case 2. Let $C_{1}=2, C_{2}=0, C_{i}=1, i=3,4,5$. We find the following group invariant system:

$$
\begin{gathered}
H_{1} \equiv I_{15}+I_{5} I_{6}+2 I_{11} I_{4}+k_{1} I_{5}^{2}+k_{2} I_{13}^{2}+k_{3} I_{14}^{2}=0, \\
H_{2} \equiv I_{9}+2 I_{1} I_{5} I_{6}-I_{3} I_{6}=0,
\end{gathered}
$$

from which we construct the Virasoro $f$-independent integrable system as follows:

$$
\begin{gathered}
p_{x t}+p_{x} r_{x}+2 r p_{x x}+k_{1} p_{x}^{2}+k_{2} p_{x y}^{2}+k_{3} r_{x y}^{2}=0, \\
p_{t}+2 x r_{x} p_{x}-p r_{x}=0 .
\end{gathered}
$$

Case 3. When choosing $C_{1}=C_{3}=1 / 2, C_{2}=1, C_{4}=C_{5}=$ $-1 / 2$, one can arrive at the following group invariant system:

$$
\begin{gathered}
H_{1} \equiv I_{9}+I_{3} I_{6}-2 I_{2} I_{6} I_{7}-I_{1} I_{5} I_{6}+k_{1} I_{7}+k_{2} I_{11}=0, \\
H_{2} \equiv I_{19}-I_{6} I_{7}-I_{4} I_{13}-2 I_{2} I_{6} I_{17}+k_{3} I_{8}+k_{4} I_{17}+k_{5} I_{23}=0 .
\end{gathered}
$$

Using the above system, we construct the corresponding Virasoro $f$-independent integrable system as follows:

$$
\begin{gathered}
p_{t}+p r_{x}-2 y p_{y} r_{x}-x p_{x} r_{x}+k_{1} p_{y}+k_{2} p_{x x}=0, \\
p_{y t}-p_{y} r_{x}-r p_{x y}-2 y p_{y y} r_{x}+k_{3} r_{y}+k_{4} p_{y y}+k_{5} p_{x x y}=0 .
\end{gathered}
$$

In the next section, we will find the group invariant solutions to the special case of the above system which reads

$$
\begin{gathered}
p_{t}+p r_{x}-2 y p_{y} r_{x}-x p_{x} r_{x}=0, \\
p_{y t}-p_{y} r_{x}-r p_{x y}-2 y p_{y y} r_{x}=0 .
\end{gathered}
$$

Case 4. Taking $C_{i}=-1, i=1,2,3,5$, and $C_{4}=-2$, we have the following group invariant system:

$$
\begin{gathered}
H_{1} \equiv I_{9}-I_{3} I_{6}+I_{4} I_{5}+I_{2} I_{6} I_{7}+k_{1} I_{8}^{2}+k_{2} I_{3}^{2}=0, \\
H_{2} \equiv I_{20}-I_{6} I_{8}-I_{4} I_{14}+I_{2} I_{6} I_{18}+k_{3} I_{3}^{2} I_{8}^{2}+k_{4} I_{3}^{2}=0,
\end{gathered}
$$

from which one can construct the Virasoro $f$-independent integrable system as follows:

$$
\begin{gathered}
p_{t}-p r_{x}+p_{x} r+y r_{x}+k_{1} r_{y}^{2}+k_{2} p^{2}=0, \\
r_{y t}+r_{x} r_{y}-r r_{x y}+y r_{x}+k_{3} r_{y}^{2}+k_{4} p^{2}=0 .
\end{gathered}
$$

\section{Group-Invariant Solutions of System (21)}

Since group-invariant solutions of nonlinear models play an important role in simulation of natural phenomena [1016], therefore we construct the group-invariant solutions to the system (21) as an example. We utilize the classical Lie symmetry group method to construct corresponding infinitesimals admitted by system (21) firstly.

Theorem 1. The symmetries of system (21) form a Lie algebra $h_{1}$ generated by the following vector fields:

$$
\begin{gathered}
V_{1}=p \partial_{p}, \quad V_{2}=y \partial_{y}, \quad V_{3}=x \partial_{p}, \\
V_{4}=\sqrt{y} \partial_{p}, \quad V_{5}=f(t) \partial_{t}-\dot{f}(t) r \partial_{r}, \\
V_{6}=g(t) x \partial_{x}+2 y g(t) \partial_{y}+g(t) p \partial_{p}+(g(t) r-\dot{g}(t) x) \partial_{r},
\end{gathered}
$$

where $f(t)$ and $g(t)$ are arbitrary functions of $t$.

We consider three special cases of functions $f(t)$ and $g(t)$.

Case 5. When $f(t)=0$, the symmetry generators of system (21) are reduced to

$$
\begin{gathered}
V_{1}=p \partial_{p}, \quad V_{2}=y \partial_{y}, \quad V_{3}=x \partial_{p}, \quad V_{4}=\sqrt{y} \partial_{p}, \\
V_{5}=g(t) x \partial_{x}+2 y g(t) \partial_{y}+g(t) p \partial_{p} \\
+(g(t) r-\dot{g}(t) x) \partial_{r} .
\end{gathered}
$$

The nonzero commutators of $V_{1}, V_{2}, V_{3}$, and $V_{4}$ are

$$
\begin{aligned}
& {\left[V_{1}, V_{3}\right]=-V_{3}, \quad\left[V_{1}, V_{4}\right]=-V_{4}, \quad\left[V_{2}, V_{4}\right]=\frac{1}{2} V_{4},} \\
& {\left[V_{3}, V_{1}\right]=V_{3}, \quad\left[V_{4}, V_{1}\right]=V_{4}, \quad\left[V_{4}, V_{2}\right]=-\frac{1}{2} V_{4} \text {. }}
\end{aligned}
$$


TABLE 1: The adjoint representation of $H_{1}$ on $h_{1}$.

\begin{tabular}{lccccc}
\hline $\operatorname{Ad}(\varepsilon \cdot)$ & $V_{1}$ & $V_{2}$ & $V_{3}$ & $V_{4}$ & $V_{5}$ \\
\hline$V_{1}$ & $V_{1}$ & $V_{2}$ & $e^{\varepsilon} V_{3}$ & $e^{\varepsilon} V_{4}$ & $V_{5}$ \\
$V_{2}$ & $V_{1}$ & $V_{2}$ & $V_{3}$ & $\cos \frac{\varepsilon}{2} V_{4}$ & $V_{5}$ \\
$V_{3}$ & $V_{1}-\varepsilon V_{3}$ & $V_{2}$ & $V_{3}$ & $V_{4}$ & $V_{5}$ \\
$V_{4}$ & $V_{1}-\varepsilon V_{4}$ & $V_{2}+\frac{\varepsilon}{2} V_{4}$ & $V_{3}$ & $V_{4}$ & $V_{5}$ \\
$V_{5}$ & $V_{1}$ & $V_{2}$ & $V_{3}$ & $V_{4}$ & $V_{5}$ \\
\hline
\end{tabular}

TABLE 2: Solutions to system (21) of Case 5.

\begin{tabular}{|c|c|c|c|}
\hline$r_{i}$ & $g(t)$ & $p(x, y, t)$ & $r(x, y, t)$ \\
\hline$r_{3}$ & 1 & $\frac{1}{\sqrt{y}}+\frac{2 C_{1}+1}{2 x}$ & $h(t) \sqrt{y}$ \\
\hline$r_{6}$ & 1 & $\pm\left(x \ln y-2 x \ln x+C_{1} x\right)$ & $k(y, t) x$ \\
\hline$r_{6}$ & 1 & $x \ln y+2 e^{\int-h(t) \mathrm{d} t} \int^{t} e^{h(a) x / \int-h(t) \mathrm{d} t} \mathrm{~d} a+e^{-\int h(t) \mathrm{d} t} F\left(e^{x / e^{\int-h(t) d t}}\right)$ & $h(t) x$ \\
\hline$r_{7}$ & 1 & 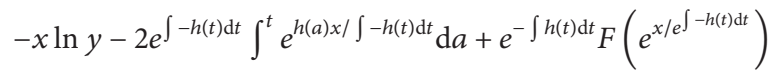 & $h(t) x$ \\
\hline$r_{13}$ & 1 & $y^{1 / \alpha} h(t)$ & $\frac{x \dot{h}(t)}{(2 / \alpha-1) h(t)}+k(y, t)$ \\
\hline$r_{3}$ & $t$ & $\frac{x \ln x}{t}+C_{1} x+C_{2} y^{1 / 2}+x h(t)$ & $\frac{-x \ln x+x}{t}+x t \dot{h}(t)+F(t) \sqrt{y}$ \\
\hline
\end{tabular}

With the help of the adjoint representation:

$$
A d(\exp (\beta V)) W=W-\beta[V, W]+\frac{\beta^{2}}{2}[V,[V, W]]-\cdots,
$$

the adjoint action of the Lie group $H_{1}$ on the Lie algebra $h_{1}$ is listed in Table 1.

Applying the method initiated by Ovsiannikov [17], we obtain the following theorem.

Theorem 2. The one-dimensional optimal system $\theta_{1}$ of $h_{1}$ is generated by

$$
\begin{gathered}
r_{1}=V_{3}, \quad r_{2}=-V_{3}, \quad r_{3}=V_{3}+V_{5}, \\
r_{4}=-V_{3}+V_{5}, \quad r_{5}=V_{2}, \quad r_{6}=V_{2}+V_{3}, \\
r_{7}=V_{2}-V_{3}, \quad r_{8}=V_{2}+\alpha V_{5}, \\
r_{9}=V_{2}+V_{3}+\alpha V_{5}, \quad r_{10}=V_{2}-V_{3}+\alpha V_{5}, \\
r_{11}=V_{1}, \quad r_{12}=V_{1}+\alpha V_{5}, \\
r_{13}=V_{1}+\alpha V_{2}, \quad r_{14}=V_{1}+\alpha V_{2}+\beta V_{5},
\end{gathered}
$$

where $\alpha, \beta$ are nonzero real constants.

Therefore, we obtain 14 nonequivalent one-dimensional subalgebras and classify the group-invariant solutions into 14 nonequivalent types. After solving the characteristic equations, we can obtain the invariants and invariant forms.
Substituting the invariant forms into system (21), we can reduce the original $(2+1)$-dimensional system to $(1+1)$ dimensional system. Since it is a tough task to find all solutions out for the every 14 nonequivalent subalgebras, we just show the results for the cases that we can deal with and list the solutions to system (21) in Table 2. Here and hereafter $h(\cdot)$, $F(\cdot)$, and $k(\cdot, \cdot)$ are arbitrary functions with respect to their variables.

Case 6. When $g(t)=0$, the symmetry generators of system (21) are reduced to

$$
\begin{array}{cl}
V_{1}=p \partial_{p}, & V_{2}=y \partial_{y}, \quad V_{3}=x \partial_{p}, \\
V_{4}=\sqrt{y} \partial_{p}, & V_{5}=f(t) \partial_{t}-\dot{f}(t) r \partial_{r} .
\end{array}
$$

In this case, the optimal system is the same as that in Theorem 2 and we can find some new solutions which are listed in Table 3.

Case 7. When $f(t)=1, g(t)=e^{t}$, the symmetry generators of system (21) are

$$
\begin{gathered}
V_{1}=p \partial_{p}, \quad V_{2}=y \partial_{y}, \quad V_{3}=x \partial_{p}, \\
V_{4}=\sqrt{y} \partial_{p}, \quad V_{5}=\partial_{t}, \\
V_{6}=e^{t}\left(x \partial_{x}+2 y \partial_{y}+p \partial_{p}+(r-x) \partial_{r}\right) .
\end{gathered}
$$


TABLE 3: Solutions to system (21) of Case 6.

\begin{tabular}{|c|c|c|c|}
\hline$r_{i}$ & $f(t)$ & $p(x, y, t)$ & $r(x, y, t)$ \\
\hline$r_{3}$ & 1 & $x t+F(x)+C_{1} \sqrt{y}+C_{2}$ & $\int \frac{x}{x \dot{F}(x)-C_{1}-F(x)} \mathrm{d} x+K(y, t)$ \\
\hline$r_{3}$ & $t$ & $x \ln t+F(x)+C_{1} \sqrt{y}+C_{2}$ & $\frac{\int\left(x /\left(x \dot{F}(x)-C_{1}-F(x)\right)\right) \mathrm{d} x}{t}+\frac{F(y)}{t}$ \\
\hline$r_{3}$ & $t^{2}$ & $-\frac{x}{t}+F(x)+C_{1} \sqrt{y}+C_{2}$ & $\frac{\int\left(x /\left(x \dot{F}(x)-C_{1}-F(x)\right)\right) \mathrm{d} x}{t^{2}}+\frac{F(y)}{t^{2}}$ \\
\hline$r_{8}$ & 1 & $C_{1} x$ & $K\left(x, y^{\alpha} e^{-t}\right)$ \\
\hline$r_{8}$ & 1 & $F(x)$ & $h\left(y^{\alpha} e^{-t}\right)$ \\
\hline$r_{8}$ & 1 & $C_{1} x+\frac{C_{2} x^{C_{3}} \sqrt{y}}{e^{t / 2 \alpha}}$ & $\frac{-x}{2 \alpha C_{3}}$ \\
\hline$r_{8}$ & 1 & $C_{1} x+C_{2}\left(y^{\alpha} e^{-t}\right)^{C_{3}}$ & $\frac{C_{3} x}{1-2 C_{3} \alpha}+h\left(y^{\alpha} e^{-t}\right)$ \\
\hline$r_{8}$ & $t$ & $C_{1} x$ & $\frac{K\left(x, y^{\alpha} e^{-t}\right)}{t}$ \\
\hline$r_{8}$ & $t$ & $F(x)$ & $\frac{h\left(y^{\alpha} e^{-t}\right)}{t}$ \\
\hline$r_{8}$ & $t$ & $C_{3} x+\frac{C_{2} x^{C_{1}} \sqrt{y}}{t^{1 / 2 a}}$ & $\frac{-x}{2 a C_{1}}$ \\
\hline$r_{8}$ & $t$ & $\frac{C_{2} C_{3} x y^{\alpha C_{1}}-t^{C_{1}}}{C_{2} y^{\alpha C_{1}}}$ & $\frac{\left(1+2 C_{1} \alpha\right) h\left(y^{\alpha} / t\right)-C_{1} x}{\left(1+2 C_{1} \alpha\right) t}$ \\
\hline$r_{8}$ & $t^{2}$ & $C_{1} x$ & $\frac{k\left(x, y e^{1 / \alpha t}\right)}{t^{2}}$ \\
\hline$r_{8}$ & $t^{2}$ & $F(x)$ & $\frac{h\left(y e^{1 / \alpha t}\right)}{t^{2}}$ \\
\hline$r_{8}$ & $t^{2}$ & $C_{1} x+C_{2} x^{C_{3}} \sqrt{y} e^{1 / 2 \alpha t}$ & $\frac{-x}{2 C_{3} \alpha t^{2}}$ \\
\hline$r_{8}$ & $t^{2}$ & $C_{1} x+C_{2}\left(y e^{1 / \alpha t}\right)^{C_{3}}$ & $\frac{-C_{3} x}{\left(2 C_{3}-1\right) \alpha t^{2}}+\frac{h\left(y e^{1 / \alpha t}\right)}{t^{2}}$ \\
\hline$r_{9}$ & 1 & $\frac{x t}{\alpha}+C_{3} x^{2}\left(y^{\alpha} e^{-t}\right)^{1 / 2 \alpha}-2 x\left(2 \ln x-C_{2}\right)$ & $\frac{-x}{4 \alpha}$ \\
\hline$r_{9}$ & 1 & $\frac{x t}{\alpha}+\left(y^{\alpha} e^{-t}\right)^{1 /\left(\alpha\left(2+C_{4}\right)\right)}+C_{3} x+C_{4} x \ln x$ & $\frac{x}{4 \alpha}+h\left(y^{\alpha} e^{-t}\right)$ \\
\hline$r_{9}$ & 1 & $\frac{x\left(\alpha \ln y-2 \alpha \ln x+C_{1} \alpha\right)}{\alpha}$ & $x h\left(y^{\alpha} e^{-t}\right)$ \\
\hline$r_{9}$ & 1 & $\frac{x t}{\alpha}+\left(h\left(y^{\alpha} e^{-t}\right)-2 \ln x\right) x$ & $\frac{-x}{2 \alpha}$ \\
\hline$r_{12}$ & 1 & $C_{3} e^{t} x^{C_{2}}\left(C_{1} x+\sqrt{y}\right)$ & $\frac{x}{C_{2}}$ \\
\hline$r_{12}$ & 1 & $h(x) e^{\alpha / t}$ & $\int \frac{h(x)}{\alpha(x \dot{h}(x)-h(x))} \mathrm{d} x+K(y, t)$ \\
\hline$r_{12}$ & $t$ & $C_{3} t x^{C_{2}}\left(C_{1} x+\sqrt{y}\right)$ & $\frac{x}{C_{2} t}$ \\
\hline$r_{12}$ & $t$ & $t^{1 / \alpha} h(x)$ & $\underline{\int h(x) /(\alpha(x \dot{h}(x)-h(x))) \mathrm{d} x+K(y, t)}$ \\
\hline
\end{tabular}


TABLE 4: Solutions to system (21) of Case 7.

\begin{tabular}{|c|c|c|}
\hline$\underline{r}_{i}$ & $p(x, y, t)$ & $r(x, y, t)$ \\
\hline$r_{3}$ & $x t+F(x)+C_{1}+C_{2} \sqrt{y}$ & $\int \frac{x}{-F(x)-C_{1}+x F^{\prime}(x)} \mathrm{d} x+h(y)$ \\
\hline$r_{4}$ & $-x t+F(x)+C_{1}+C_{2} \sqrt{y}$ & $\int \frac{-x}{-F(x)-C_{1}+x F^{\prime}(x)} \mathrm{d} x+h(y)$ \\
\hline$r_{8}$ & $C_{1} x$ & $K\left(x, \frac{y}{e^{t / \alpha}}\right)$ \\
\hline$r_{8}$ & $F(x)$ & $h\left(\frac{y}{e^{t / \alpha}}\right)$ \\
\hline$r_{8}$ & $C_{3} x+C_{2} x_{1}^{C} \frac{\sqrt{y}}{e^{t / 2 \alpha}}$ & $\frac{-x}{2 \alpha C_{1}}$ \\
\hline$r_{8}$ & $C_{3} x+C_{2} \frac{y^{C_{1}}}{e^{\left(C_{1} t\right) / \alpha}}$ & $\frac{C_{1} x}{\alpha\left(1-2 C_{1}\right)}+F\left(\frac{y}{e^{t / \alpha}}\right)$ \\
\hline$r_{9}$ & $\frac{x t}{\alpha}+F(x)$ & $\frac{x}{\alpha\left(-F(x)+x F^{\prime}(x)\right)} \mathrm{d} x+h\left(\frac{y}{e^{t / \alpha}}\right)$ \\
\hline$r_{9}$ & $\frac{x t}{\alpha}-2 x \ln \left(C_{3} x\right)+C_{1} x+C_{3} x \sqrt{\frac{y}{e^{t / \alpha}}}$ & $\frac{-x}{2 \alpha}$ \\
\hline$r_{9}$ & $\frac{x t}{\alpha}+\left(\left(\left(C_{2}-2\right) \ln x+C_{1}\right) x e^{C_{4} / C_{2}}-\left(\frac{y}{e^{t / \alpha}}\right)^{1 / C_{2}}\right) e^{-C_{4} / C_{2}}$ & $\frac{x}{\alpha\left(C_{2}-2\right)}+F\left(\frac{y}{e^{t / \alpha}}\right)$ \\
\hline & $\frac{x t}{\alpha}+x\left(\ln \left(\frac{y}{e^{t / \alpha}}\right)+C_{1}-2 \ln x\right)$ & $x F\left(\frac{y}{e^{t / \alpha}}\right)$ \\
\hline$r_{10}$ & $\frac{-x t}{\alpha}+F(x)$ & $\frac{-x}{\alpha\left(-F(x)+x F^{\prime}(x)\right)} \mathrm{d} x+h\left(\frac{y}{e^{t / \alpha}}\right)$ \\
\hline$r_{10}$ & $\frac{-x t}{\alpha}+2 x \ln \left(C_{3} x\right)+C_{1} x+C_{3} x \sqrt{\frac{y}{e^{t / \alpha}}}$ & $\frac{-x}{2 \alpha}$ \\
\hline$r_{10}$ & $\frac{-x t}{\alpha}+\left(\left(\left(-C_{2}+2\right) \ln x+C_{1}\right) x e^{C_{4} / C_{2}}-\left(\frac{y}{e^{t / \alpha}}\right)^{1 / C_{2}}\right) e^{-C_{4} / C_{2}}$ & $\frac{x}{\alpha\left(C_{2}-2\right)}+F\left(\frac{y}{e^{t / \alpha}}\right)$ \\
\hline$r_{10}$ & $\frac{-x t}{\alpha}+x\left(-\ln \left(\frac{y}{e^{t / \alpha}}\right)+C_{1}+2 \ln x\right)$ & $x F\left(\frac{y}{e^{t / \alpha}}\right)$ \\
\hline$r_{12}$ & $e^{t / \alpha}+C_{1} x+C_{2} \sqrt{y}$ & $h(y)+\frac{x}{\alpha}$ \\
\hline$r_{14}$ & $y^{1 / \alpha} F(x)\left(\frac{y}{e^{\alpha t / \beta}}\right)^{-1 / \alpha}$ & $\int \frac{F(x)}{\beta\left(x F^{\prime}(x)-F(x)\right)} \mathrm{d} x+h\left(\frac{y}{e^{\alpha t / \beta}}\right)$ \\
\hline$r_{14}$ & $y^{1 / \alpha}\left(x^{(\alpha / 2-1)} C_{1}\left(C_{3} x^{C_{2}}\right)^{(1-\alpha / 2)}\left(\frac{y}{e^{\alpha t / \beta}}\right)^{\alpha-2 / 2 \alpha}+C_{3} x^{C_{2}}\left(\frac{y}{e^{\alpha t / \beta}}\right)^{-1 / \alpha}\right)$ & $\frac{x}{\beta\left(C_{2}-1\right)}$ \\
\hline$r_{14}$ & $C_{1} y^{1 / \alpha}$ & $F\left(\frac{y}{e^{\alpha t / \beta}}\right)$ \\
\hline
\end{tabular}

By simple calculation, we obtain that the optimal system in this case is the same as that in Theorem 2. And we list the new solutions in Table 4.

\section{Concluding Remarks}

In this paper, we extend the Virasoro-type symmetry prolongation approach from single equations to coupled systems of two-component nonlinear equations. Four types of new nonlinear Virasoro integrable systems are constructed. Furthermore, we obtain the one-dimensional optimal system and group-invariant solutions to one of the model systems, namely, system (21).

\section{Conflict of Interests}

The authors declare that there is no conflict of interests regarding the publication of this paper. 


\section{Acknowledgments}

Parts of this research were done when Lizhen Wang was visiting the Institute of Mathematical Sciences (IMS) of the Chinese University of Hong Kong. This work is supported by the National Natural Science Foundations of China (Grant nos. 11201371, 11371323, 11101332, and 11071195), the National Natural Science Foundation of Shaanxi Province (Grant no. 2012JQ1013), and the Program of Shaanxi Provincial Department of Education (Grant no. 11JK0482).

\section{References}

[1] A. Maccari, "A new integrable Davey-Stewartson-type equation," Journal of Mathematical Physics, vol. 40, no. 8, pp. 39713977, 1999.

[2] F. Calogero and W. Eckhaus, "Nonlinear evolution equations, rescalings, model PDEs and their integrability. II," Inverse Problems, vol. 4, no. 1, pp. 11-33, 1988.

[3] S.-Y. Lou and X.-B. Hu, "Infinitely many Lax pairs and symmetry constraints of the KP equation," Journal of Mathematical Physics, vol. 38, no. 12, pp. 6401-6427, 1997.

[4] S.-y. Lou, "Searching for higher-dimensional integrable models from lower ones via Painlevé analysis," Physical Review Letters, vol. 80, no. 23, pp. 5027-5031, 1998.

[5] S. Y. Lou and X. B. Hu, "Infinitely many symmetries of the Davey-Stewartson equation," Journal of Physics A, vol. 27, no. 7, pp. L207-L212, 1994.

[6] S. Y. Lou, J. Yu, and J. Lin, " $(2+1)$-dimensional models with Virasoro-type symmetry algebra," Journal of Physics A, vol. 28, no. 6, pp. L191-L196, 1995.

[7] J. Lin, S.-y. Lou, and K. Wang, "High-dimensional Virasoro integrable models and exact solutions," Physics Letters A, vol. 287, no. 3-4, pp. 257-267, 2001.

[8] S. F. Shen, "Virasoro symmetry subalgebra, multi-linear variable separation solutions and localized excitations of higher-dimensional differential-difference models," Acta Physica Sinica, vol. 55, no. 11, pp. 5606-5610, 2006.

[9] L.-Z. Wang, Q. Huang, S.-F. Shen, and W. Gao, "Some new (2+1)-dimensional integrable systems with infinitely dimensional Virasoro-type symmetry algebra," Acta Mathematicae Applicatae Sinica, vol. 36, no. 6, pp. 1000-1007, 2013 (Chinese).

[10] K.-S. Chou, G.-X. Li, and C. Qu, "A note on optimal systems for the heat equation," Journal of Mathematical Analysis and Applications, vol. 261, no. 2, pp. 741-751, 2001.

[11] K.-S. Chou and G.-X. Li, "Optimal systems and invariant solutions for the curve shortening problem," Communications in Analysis and Geometry, vol. 10, no. 2, pp. 241-274, 2002.

[12] C.-Z. Qu and Q. Huang, "Symmetry reductions and exact solutions of the affine heat equation," Journal of Mathematical Analysis and Applications, vol. 346, no. 2, pp. 521-530, 2008.

[13] D. K. Ludlow, P. A. Clarkson, and A. P. Bassom, "Nonclassical symmetry reductions of the three-dimensional incompressible Navier-Stokes equations," Journal of Physics A, vol. 31, no. 39, pp. 7965-7980, 1998.

[14] D. K. Ludlow, P. A. Clarkson, and A. P. Bassom, "Similarity reductions and exact solutions for the two-dimensional incompressible Navier-Stokes equations," Studies in Applied Mathematics, vol. 103, no. 3, pp. 183-240, 1999.
[15] L.-Z. Wang, M. Gou, and C.-Z. Qu, “Conditional Lie Bäcklund symmetries of Hamilton-Jacobi equations," Chinese Physics Letters, vol. 24, no. 12, pp. 3293-3296, 2007.

[16] L.-Z. Wang and Q. Huang, "Symmetries and group-invariant solutions for transonic pressure-gradient equations," Communications in Theoretical Physics, vol. 56, no. 2, pp. 199-206, 2011.

[17] L. V. Ovsiannikov, Group Analysis of Differential Equations, Academic Press, New York, NY, USA, 1982. 


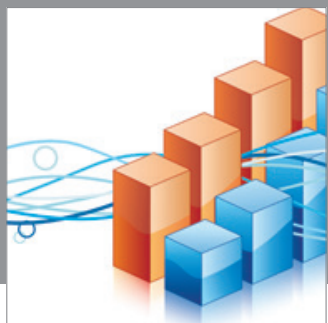

Advances in

Operations Research

mansans

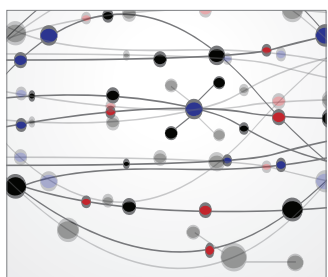

The Scientific World Journal
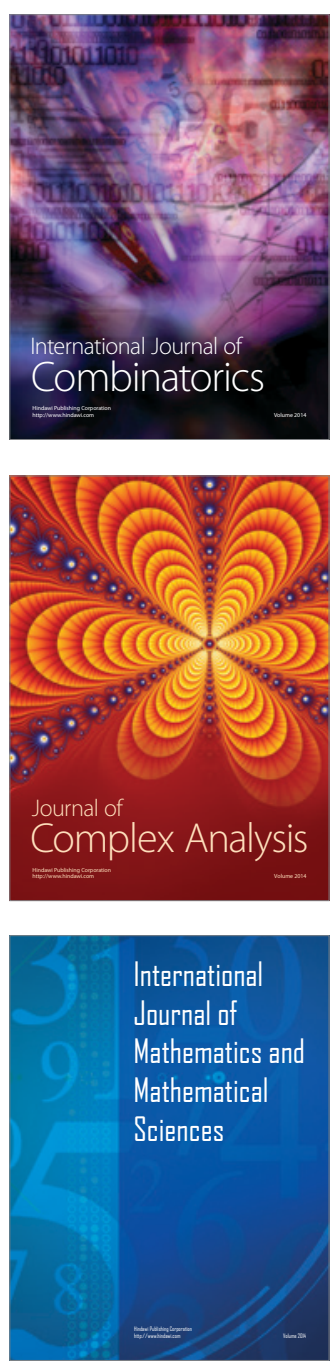
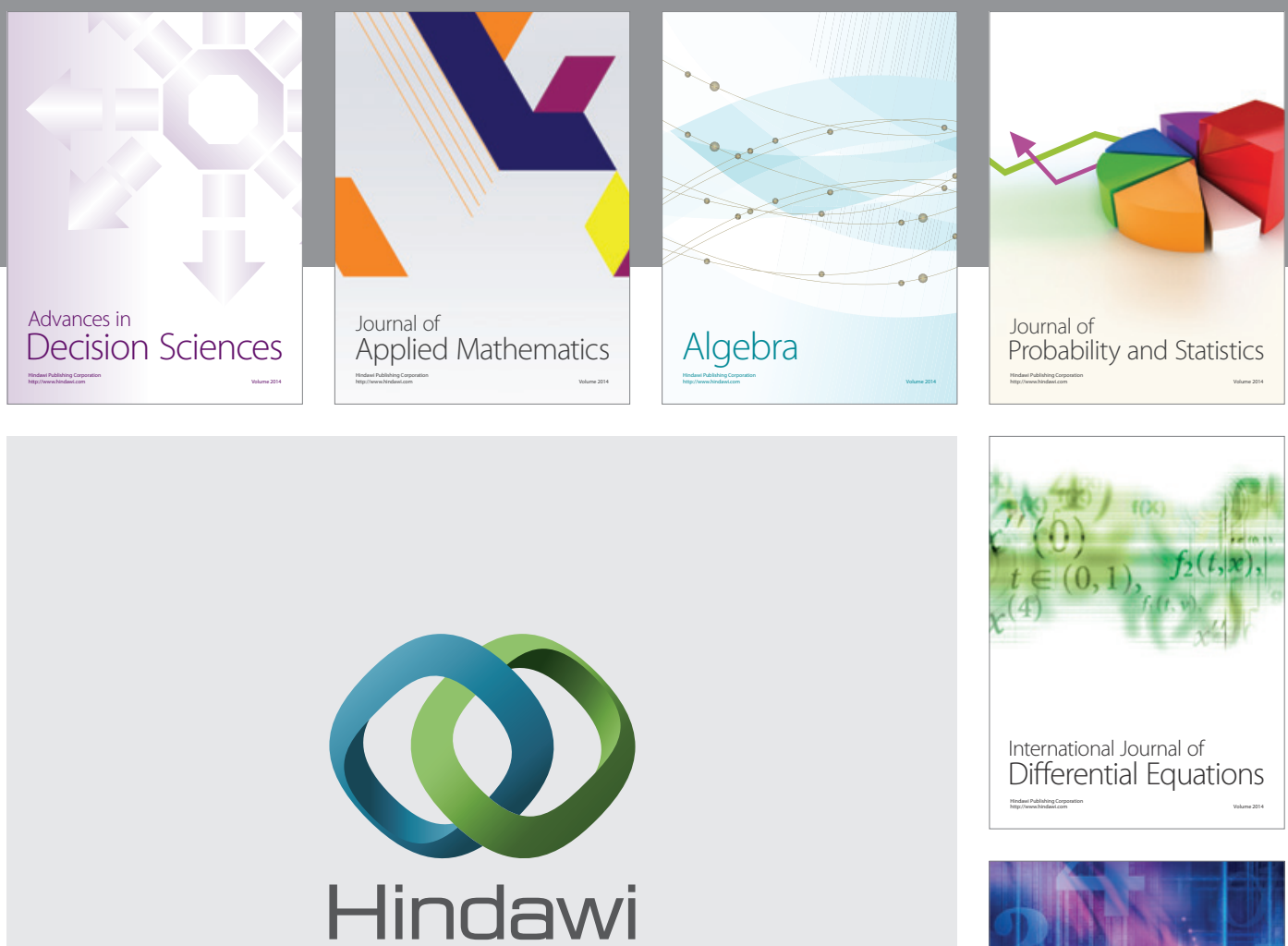

Submit your manuscripts at http://www.hindawi.com
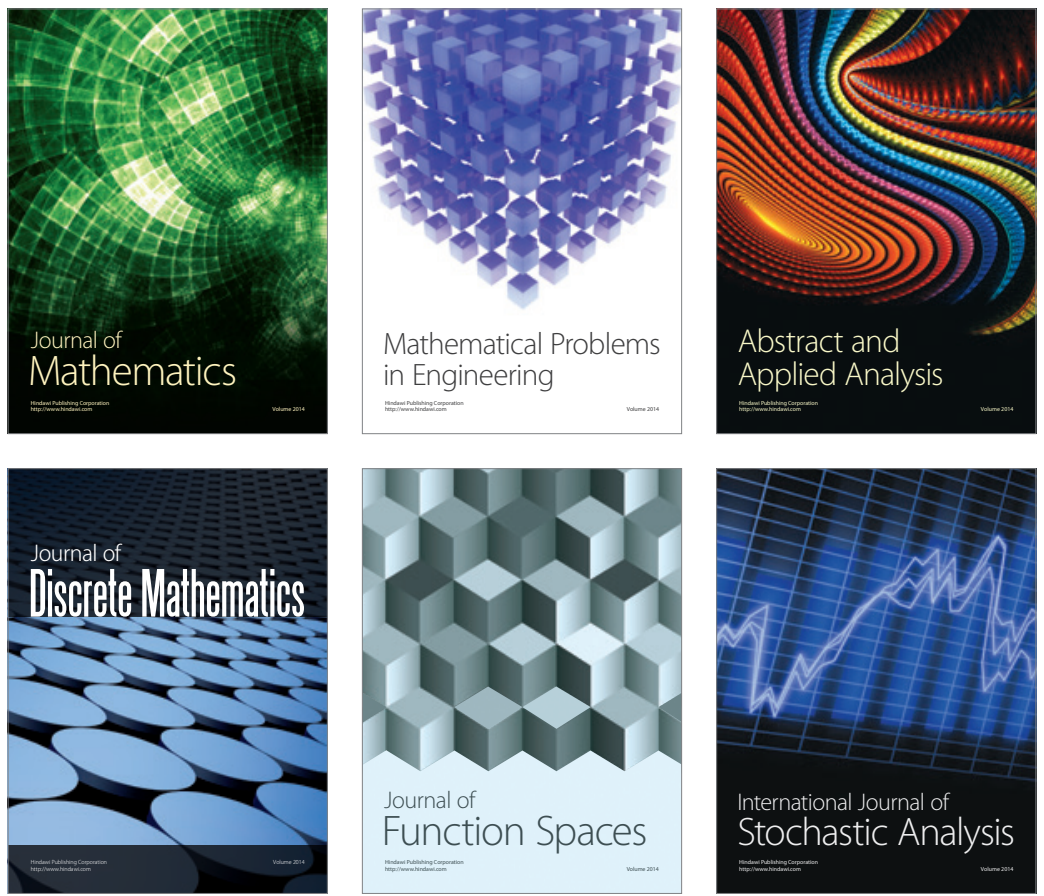

Journal of

Function Spaces

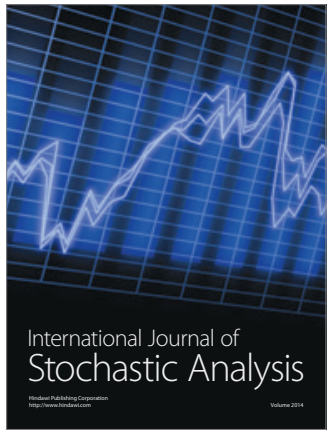

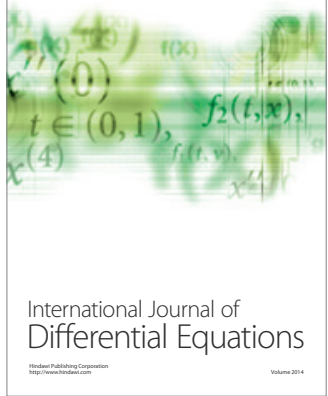
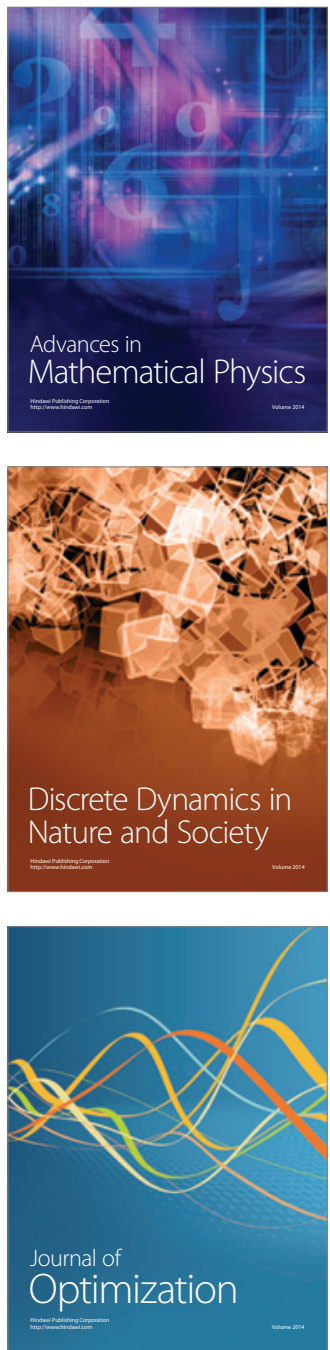